
ПРИ ЭНДОКРИННЫХ ЗАБОЛЕВАНИЯХ

\author{
() Е.А. Пигарова*, Л.К. Дзеранова
}

Национальный медицинский исследовательский центр эндокринологии, Москва, Россия

Обоснование. Дефицит и недостаточность витамина D являются широко распространенными лабораторными отклонениями в медицине, но их частота при эндокринных заболеваниях в целом не изучена.

Цель. Целью данного исследования было провести сравнительную оценку уровня витамина D у пациентов с сахарным диабетом 2 типа, первичным гиперпаратиреозом (ПГПТ), центральным гиперкортицизмом (болезнью ИценкоКушинга) и акромегалией.

Материалы и методы. Общий 25(OH)D определяли иммунохемилюминесцентным методом (лаборатория участвует в программе DEQAS). Все пациенты имели скорость клубочковой фильтрации >60 мл/мин, не принимали добавки витамина D в течение предыдущего месяца.

Результаты. В исследование были включены 365 пациентов, которые после применения критериев включения/ исключения были разделены на основные группы исследования: 33 пациента с сахарным диабетом 2 типа, 23 пациента с ПГПТ, 68 пациентов с болезнью Иценко-Кушинга, 22 пациента с акромегалией и 141 практически здоровый пациент. Значимо более низкие уровни витамина D были обнаружены у пациентов с сахарным диабетом (14,8 нг/мл), акромегалией (14,9 нг/мл), болезнью Иценко-Кушинга (14,6 нг/мл) и ПГПТ (15,9нг/мл) по сравнению с группой здоровых пациентов (18,8 нг/мл).

Заключение. Результаты исследования демонстрируют высокую распространенность дефицита витамина D не только в группах пациентов с хроническими заболеваниями, но и среди практически здоровых пациентов. Необходимы дальнейшие исследования для устранения причин высокого дефицита витамина D при описанных эндокринных заболеваниях.

КЛЮЧЕВЫЕ СЛОВА: витамин D; 25(ОН)D; холекальциферол; паратгормон; ПТГ; акромегалия; болезнь Иценко-Кушинга; сахарный диабет 2 mипа; первичный гиперпаратиреоз.

\title{
HIGH PREVALENCE OF LOW VITAMIN D LEVELS IN ENDOCRINE DISORDERS
}

(c) Ekaterina A. Pigarova, Larisa K. Dzeranova

Endocrinology Research Centre, Moscow, Russia

BACKGROUND: Vitamin D deficiency and insufficiency are widespread medical abnormalities, but their frequency in endocrine diseases has generally not been studied.

AIM: To provide a comparative assessment of vitamin D levels in patients with diabetes mellitus type 2, primary hyperparathyroidism (PHPT), central hypercortisolism (Cushing's disease; CD) and acromegaly.

MATERIALS AND METHODS: Total 25(OH)D was determined using the immunochemiluminescent method (the laboratory participates in the DEQAS program). All patients had GFR $>60 \mathrm{ml} / \mathrm{min}$, no history of use of vitamin D supplementation within previous month.

RESULTS: The study included 365 patients who, after applying the inclusion/exclusion criteria, were divided into 5 main research groups: 33 patients with diabetes mellitus type 2, 23 patients with PHPT, 68 patients with CD, 22 patients with acromegaly, and 141 apparently healthy patients. Significantly low levels of vitamin D were found in patients with diabetes mellitus type $2(14.8 \mathrm{ng} / \mathrm{ml})$, acromegaly $(14.9 \mathrm{ng} / \mathrm{ml})$, CD $(14.6 \mathrm{ng} / \mathrm{ml})$, and PHPT $(15.9 \mathrm{ng} / \mathrm{ml})$ compared with a group of otherwise healthy patients $(18.8 \mathrm{ng} / \mathrm{ml})$.

CONCLUSION: The results of the study demonstrate a high prevalence of vitamin D deficiency not only in groups of patients with chronic diseases, but also among practically healthy patients. Further studies are needed to address the causes of high vitamin $\mathrm{D}$ deficiency in the described endocrine diseases.

KEYWORDS: vitamin D; 25(OH)D; cholecalciferol; parathyroid hormone; PTH; acromegaly; Cushing's disease; type 2 diabetes mellitus; primary hyperparathyroidism. 


\section{ОБОСНОВАНИЕ}

Роль витамина D в здоровье костей хорошо известна, поскольку дефицит витамина D является причиной большинства случаев рахита и остеомаляции, а также ускоряет потерю костной массы и развитие остеопороза у пожилых людей. Доклинические данные свидетельствуют о том, что недостаточность витамина D может иметь внескелетные эффекты и предрасполагать ко многим заболеваниям [1].

Витамин D синтезируется в коже под воздействием ультрафиолетовых лучей солнца, также его получают из пищи, особенно жирной рыбы. Для активации он проходит два гидроксилирования: первое - в печени, до 25-гидроксивитамина D (25(OH)D), второе - в почках, до 1,25-дигидроксивитамина D $(1,25(\mathrm{OH})$ D). Активная форма витамина $\mathrm{D}$, кальцитриол $\left(1,25(\mathrm{OH})_{2} \mathrm{D}\right)$, является по всем своим характеристикам стероидным гормоном. Кальцитриол может проникать в клетку, связываться с рецептором к витамину D (VDR), а затем со специфическими регуляторными участками генома. Производство 1,25(OH) 2 D стимулируется паратиреоидным гормоном (ПТГ), подавляется кальцием и фактором роста фибробластов 23 (FGF23) [2].

Экстраренальный синтез 1,25(OH) 2 п происходит под влиянием цитокинов и важен для паракринной регуляции различных процессов в организме. Биохимические и генетические данные ясно демонстрируют, что эндокринная система витамина D может регулировать порядка 300 генов в организме, при этом в основном гены, не связанные с кальциевым или костным гомеостазом, а участвующие в пролиферации и дифференцировке клеток, влияющих на функции иммунной, мышечной, сердечно-сосудистой и других систем [3]. В то же время на метаболизм витамина D оказывают влияние многие гормональные факторы, что может иметь патогенетическое значение в развитии как самих этих заболеваний, так и осложнений [4].

\section{ЦЕЛЬ ИССЛЕДОВАНИЯ}

Цель данного исследования - изучить статус витамина D и параметры кальций-фосфорного обмена у пациентов с эндокринными заболеваниями.

\section{МАТЕРИАЛЫ И МЕТОДЫ}

\section{Место и время проведения исследования}

Место проведения. Исследование проведено на базе ФГБУ «НМИЦ эндокринологии» Минздрава России.

Время исследования. Сентябрь-октябрь 2014 г.

Изучаемые популяции (одна или несколько)

В исследование включены пациенты, которым определялись 25(OH)D и ПтГ по данным лабораторной системы учреждения в сентябре и октябре 2014 г. $(\mathrm{n}=365)$. Пациенты были разделены по группам исследования, согласно критериям включения и исключения.

\section{Популяция 1.}

Критерии включения: сахарный диабет 2 типа.

Критерии исключения: первичный гиперпаратиреоз (ПГПТ), болезнь Иценко-Кушинга, акромегалия.
Популяция 2.

Критерии включения: ПГПТ.

Критерии исключения: сахарный диабет 2 типа, болезнь Иценко-Кушинга, акромегалия.

\section{Популяция 3.}

Критерии включения: болезнь Иценко-Кушинга.

Критерии исключения: сахарный диабет 2 типа, ПГПТ, акромегалия.

\section{Популяция 4.}

Критерии включения: акромегалия.

Критерии исключения: сахарный диабет 2 типа, ПГПт, болезнь Иценко-Кушинга.

\section{Популячия 5: практически здоровые пачиенты (контроль).}

Критерии исключения: акромегалия, сахарный диабет 2 типа, ПГПТ, болезнь Иценко-Кушинга.

Критерии исключения, общие для всех популячий: 1) уровень креатинина крови более 100 мкмоль/л; 2) скорость клубочковой фильтрации менее 60 мл/мин/1,73 м²; 3) прием препаратов активных метаболитов витамина D (альфакальцидол, кальцитриол, парикальцитол) в течение 1 мес до взятия анализа крови; 4) применение препаратов лечения остеопороза (стронция ранелат, деносумаб, золедроновая кислота, ибандроновая кислота, алендроновая кислота).

Способ формирования выборки из изучаемой популяции (или нескольких выборок из нескольких изучаемых популяций)

Формирование выборки проводилось сплошным методом.

\section{Дизайн исследования}

Одноцентровое поперечное наблюдательное сравнительное нерандомизированное исследование.

\section{Методы}

Клинические данные пациентов, критерии включения и исключения были проанализированы из электронной истории болезни и выписных эпикризов. Лабораторное исследование проводилось следующими методами: общий 25(OH)D - иммунохемилюминесцентный метод, участвующий в программе внешнего контроля качества определения DEQAS (Liason DiaSorin, Франция), ПТГ, остеокальцин, С-терминальный телопептид коллагена 1 типа - иммуноэлектрохемилюминесцентный метод (ELECSYS Roche, Франция), общий кальций, ионизированный кальций, креатинин, фосфор - на автоматическом биохимическом анализаторе (ARCHITECT c8000 Abbott, США) с помощью коммерческих наборов.

\section{Статистический анализ}

Обработка данных проводилась с помощью применения пакета программ Statistica v.13 (Statsoft, США). Для представления данных использовались медиана (Me), а также 1-й и 3-й квартили (Q1; Q3). Для оценки корреляции между параметрами использовался коэффициент ранговой корреляции Спирмена. Для выявления различий 2 групп по количественным признакам при- 


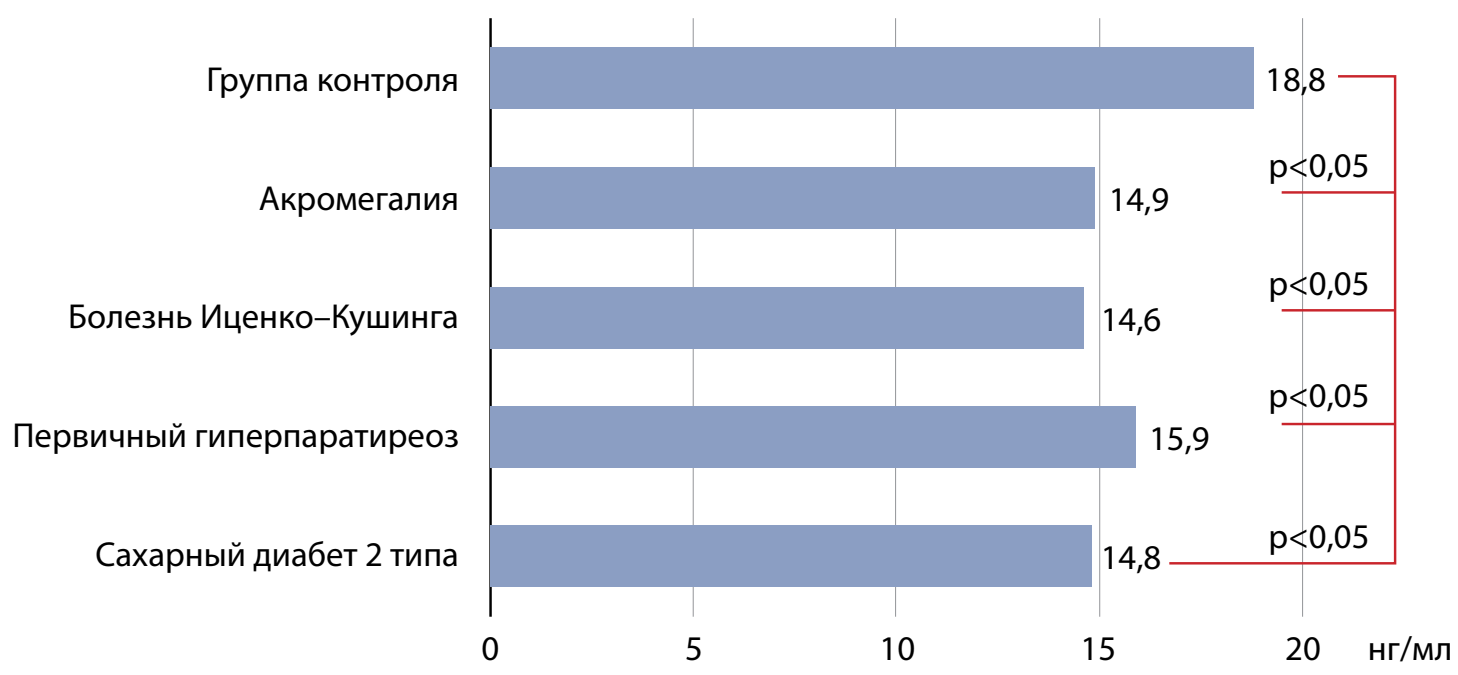

Рисунок 1. Сравнение уровней 25(OH)D сыворотки крови у пациентов с акромегалией, болезнью Иценко-Кушинга, первичным гиперпаратиреозом, сахарным диабетом 2 типа и группы контроля (U-тест Манна-Уитни).

менялся U-критерий Манна-Уитни, по качественным двусторонний критерий Фишера. Уровень статистической значимости принимался за $\mathrm{p}<0,05$.

\section{Этическая экспертиза}

Дизайн исследования одобрен локальным этическим комитетом ФГБУ «НМИЦ эндокринологии» Минздрава России, протокол №22 от 27.10.2021.

\section{РЕЗУЛЬТАТЬ}

Характеристика включенных в исследование пациентов представлена в таблице 1. Не было получено различий между группами по половому составу ( $>>0,05)$.

Статус витамина D у пациентов с эндокринными заболеваниями и группы контроля

По данным исследования, в группе контроля медиана уровня общего 25(OH)D в сыворотке крови составила 18,8 нг/мл. У пациентов с эндокринными заболеваниями уровни 25(OH)D были статистически значимо ниже, чем в группе контроля (рис. 1) - для всех попарных сравнений с учетом поправки Бонферрони $\mathrm{p}<0,05$.

Частота выраженного дефицита (0-9,9 нг/мл), дефицита (10-19,9 нг/мл), недостаточности (20-29,9 нг/мл) и адекватных уровней 25(OH)D (30-100 нг/мл) сыворотки крови у пациентов с акромегалией, болезнью Иценко-Кушинга, первичным гиперпаратиреозом, сахарным диабетом 2 типа и группы контроля представлена на рисунке 2.

Состояние кальций-фосфорного обмена в группе контроля

У пациентов из группы контроля отмечено повышение концентрации ПТГ у 29,8\% (42/141) пациентов, что отражает декомпенсацию кальций-фосфорного обмена с развитием вторичного гиперпаратиреоза. Корреляционный анализ по Спирмену показал наличие обратной зависимости 25(OH)D vs ПTГ ( $r=-0,31 ; p=0,0002)$, а также для 25(OH)D vs фосфор ( $r=-0,34 ; p=0,02)$, и прямую зависимость для 25(OH)D vs C-терминальный телопептид коллагена 1 типа (CTX) $(r=0,51 ; p=0,03)$.

Состояние кальций-фосфорного обмена в группе пациентов с сахарным диабетом 2 типа

У 18,2\% (6/33) пациентов с сахарным диабетом 2 типа выявлено повышение концентрации ПтГ. При сравнении с группой контроля частота повышения ПТГ статистически значимо не отличалась (p>0,05). При этом корреляционный анализ по Спирмену показал наличие обратной зависимости 25(OH)D vs ПTГ ( $r=-0,60 ; p=0,0002)$, а также прямую зависимость для 25(OH)D vs остеокальцин $(r=0,37 ; p=0,04)$.

Таблица 1. Демографические и клинико-анамнестические характеристики пациентов исследования

\begin{tabular}{|c|c|c|c|c|c|}
\hline Характеристика & $\begin{array}{c}\text { Сахарный } \\
\text { диабет } 2 \text { типа }\end{array}$ & $\begin{array}{l}\text { Первичный ги- } \\
\text { перпаратиреоз }\end{array}$ & $\begin{array}{l}\text { Болезнь } \\
\text { Иценко- } \\
\text { Кушинга }\end{array}$ & Акромегалия & Контроль \\
\hline $\begin{array}{l}\text { Количество человек } \\
\text { в группе }\end{array}$ & $n=33$ & $n=23$ & $n=68$ & $n=22$ & $n=141$ \\
\hline \multicolumn{6}{|l|}{ Пол: } \\
\hline мужской & $5(15 \%)$ & $2(9 \%)$ & 13 (19\%) & $2(9 \%)$ & $24(17 \%)$ \\
\hline женский & $28(85 \%)$ & $21(91 \%)$ & $55(81 \%)$ & $20(91 \%)$ & $117(83 \%)$ \\
\hline $\begin{array}{l}\text { Активная стадия } \\
\text { заболевания }\end{array}$ & $33(100 \%)$ & $23(100 \%)$ & 27 (40\%) & $22(100 \%)$ & - \\
\hline Возраст, лет & $60[55 ; 67]$ & $58[44 ; 65]$ & $41[31 ; 52]$ & $51[45,5 ; 59,5]$ & $54[35 ; 63]$ \\
\hline
\end{tabular}




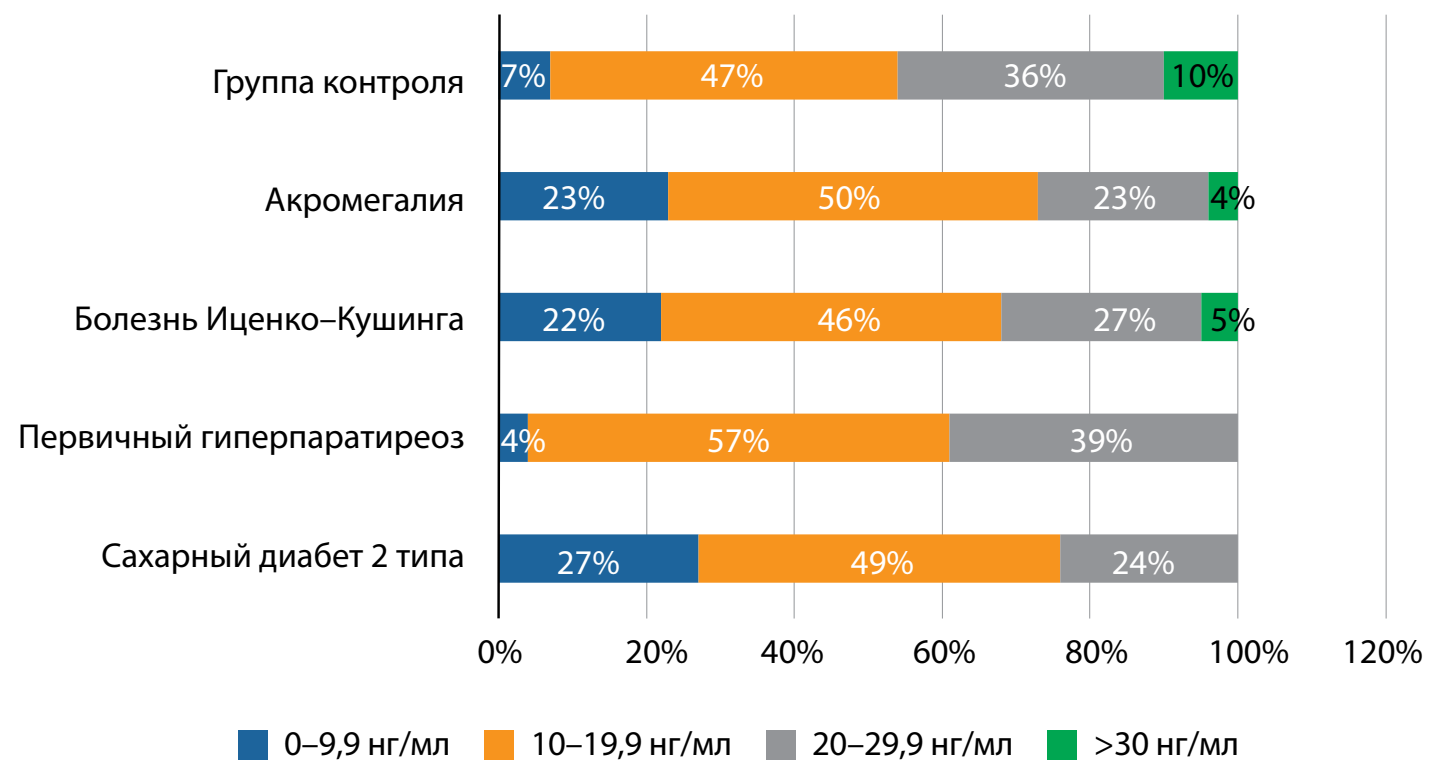

Рисунок 2. Частота выраженного дефицита (0-9,9 нг/мл), дефицита (10-19,9 нг/мл), недостаточности (20-29,9 нг/мл) и адекватных уровней 25(OH)D (30-100 нг/мл) сыворотки крови у пациентов с акромегалией, болезнью Иценко-Кушинга, первичным гиперпаратиреозом, сахарным диабетом 2 типа и группы контроля.

Таблица 2. Параметры кальций-фосфорного обмена и маркеры костного метаболизма у пациентов с акромегалией, болезнью Иценко-Кушинга, первичным гиперпаратиреозом, сахарным диабетом 2 типа и группы контроля

\begin{tabular}{|c|c|c|c|c|c|}
\hline & $\begin{array}{c}\text { Сахарный } \\
\text { диабет } 2 \text { типа } \\
\text { (n=33) }\end{array}$ & $\begin{array}{c}\text { Первичный ги- } \\
\text { перпаратиреоз } \\
\text { (n=23) }\end{array}$ & $\begin{array}{c}\text { Болезнь } \\
\text { Иценко- } \\
\text { Кушинга }(\mathrm{n}=68)\end{array}$ & $\begin{array}{c}\text { Акромегалия } \\
(n=22)\end{array}$ & $\begin{array}{c}\text { Контроль } \\
(n=141)\end{array}$ \\
\hline 25(OH)D, нг/мл & $\begin{array}{c}14,8 \\
{[9,3 ; 18,5]}\end{array}$ & $\begin{array}{c}15,9 \\
{[13 ; 24,6]}\end{array}$ & $\begin{array}{c}14,6 \\
{[10,5 ; 21,5]}\end{array}$ & $\begin{array}{c}14,9 \\
{[12,2 ; 21,2]}\end{array}$ & $\begin{array}{c}18,8 \\
{[14,1 ; 24,6]}\end{array}$ \\
\hline ПТГ, пг/мл & $\begin{array}{c}44,2 \\
{[30,1 ; 56,8]}\end{array}$ & $\begin{array}{c}100,7 \\
{[77,4 ; 145,2]}\end{array}$ & $\begin{array}{c}39,7 \\
{[31,0 ; 52,0]}\end{array}$ & $\begin{array}{c}40,8 \\
{[30,7 ; 52,1]}\end{array}$ & $\begin{array}{c}46,5 \\
{[32,7 ; 69]}\end{array}$ \\
\hline $\begin{array}{l}\text { Общий кальций, } \\
\text { ммоль/л }\end{array}$ & $\begin{array}{c}2,38 \\
{[2,34 ; 2,5]}\end{array}$ & $\begin{array}{c}2,6 \\
{[2,5 ; 2,74]}\end{array}$ & $\begin{array}{c}2,37 \\
{[2,3 ; 2,44]}\end{array}$ & $\begin{array}{c}2,36 \\
{[2,30 ; 2,46]}\end{array}$ & $\begin{array}{c}2,33 \\
{[2,26 ; 2,46]}\end{array}$ \\
\hline $\begin{array}{l}\text { Ионизированный } \\
\text { кальций, ммоль/л }\end{array}$ & $\begin{array}{c}1,14 \\
{[1,12 ; 1,19]}\end{array}$ & $\begin{array}{c}1,22 \\
{[1,15 ; 1,29]}\end{array}$ & $\begin{array}{c}1,14 \\
{[1,1 ; 1,17]}\end{array}$ & $\begin{array}{c}1,11 \\
{[1,07 ; 1,14]}\end{array}$ & $\begin{array}{c}1,11 \\
{[1,07 ; 1,15]}\end{array}$ \\
\hline Фосфор, ммоль/л & $\begin{array}{c}1,16 \\
{[0,98 ; 1,22]}\end{array}$ & $\begin{array}{c}0,86 \\
{[0,80 ; 0,92]}\end{array}$ & $\begin{array}{c}1,10 \\
{[1,0 ; 1,25]}\end{array}$ & $\begin{array}{c}1,29 \\
{[1,27 ; 1,41]}\end{array}$ & $\begin{array}{c}1,11 \\
{[0,99 ; 1,28]}\end{array}$ \\
\hline Остеокальцин, нг/мл & $\begin{array}{c}22,5 \\
{[11,9 ; 33,1]}\end{array}$ & $\begin{array}{c}28,8 \\
{[21,9 ; 43,4]}\end{array}$ & $\begin{array}{c}10,8 \\
{[6,2 ; 20,5]}\end{array}$ & $\begin{array}{c}27,5 \\
{[23,1 ; 33,8]}\end{array}$ & $\begin{array}{c}19,7 \\
{[17,1 ; 29,6]}\end{array}$ \\
\hline $\begin{array}{l}\text { С-концевой } \\
\text { телопептид коллагена } \\
1 \text { типа (СТX), нг/мл }\end{array}$ & $\begin{array}{c}0,405 \\
{[0,310 ; 0,454]}\end{array}$ & $\begin{array}{c}0,423 \\
{[0,386 ; 0,705]}\end{array}$ & $\begin{array}{c}0,473 \\
{[0,200 ; 0,620]}\end{array}$ & $\begin{array}{c}0,620 \\
{[0,500 ; 0,660]}\end{array}$ & $\begin{array}{c}0,530 \\
{[0,467 ; 0,722]}\end{array}$ \\
\hline
\end{tabular}

Состояние кальций-фосфорного обмена в группе пациентов с первичным гиперпаратиреозом

У пациентов с ПГПТ в 100\% случаев отмечено повышение уровня ПТГ, что являлось определяющим в постановке диагноза. При проведении корреляционного анализа по Спирмену показано отсутствие каких-либо зависимостей для 25(OH)D относительно изучавшихся маркеров. Тогда как ПТГ был связан отрицательной зависимостью по отношению к уровню фосфора крови $(r=-0,64 ; p=0,02)$ и положительной по отношению к уровню общего кальция крови $(r=0,48 ; \mathrm{p}=0,03)$ и СТХ $(r=0,66, p=0,02)$.
Состояние кальций-фосфорного обмена в группе пациентов с болезнью Иценко-Кушинга

При болезни Иценко-Кушинга вторичный гиперпаратиреоз выявлен у 23,5\% (16/68). При сравнении с группой контроля частота повышения ПТГ статистически значимо не отличалась ( $>0,05)$. При этом корреляционный анализ по Спирмену показал наличие обратной зависимости 25(OH)D vs ПТГ ( $r=-0,31 ; p=0,01)$ и ПТГ vs фосфор $(r=-0,30 ; p=0,049)$.

В активной фазе болезни, при наличии высоких концентраций кортизола в крови, пациенты отличались еще более низкими уровнями $25(\mathrm{OH}) \mathrm{D}-13,5[9,57 ; 17,5]$ 
vs $20,2[11,5 ; 26,1]$ нг/мл ( лее высокими уровнями общего кальция крови - 2,39 $[2,32 ; 2,44]$ vs $2,3[2,24 ; 2,4]$ ммоль/л $(p=0,008)$.

\section{Состояние кальций-фосфорного обмена в группе} пациентов с акромегалией

У пациентов с акромегалией вторичный гиперпаратиреоз встречался в 9\% (2/22) случаев. При сравнении с группой контроля частота повышения ПтГ статистически значимо не отличалась ( $>>0,05)$. При проведении корреляционного анализа по Спирмену показано наличие обратной зависимости 25(OH)D vs ПТГ ( $r=-0,49 ; p=0,02)$, без выявления других взаимосвязей 25(OH)D или ПТГ с другими параметрами.

\section{ОБСУЖДЕНИЕ}

\section{Репрезентативность выборок}

Репрезентативность выборки пациентов с болезнью Иценко-Кушинга является несколько ограниченной, что определяется уменьшенной долей пациентов, имеющих активную стадию заболевания. Также в исследовании не проводился анализ множественных лекарственных факторов, помимо препаратов витамина D, которые потенциально могут приводить к нарушению всасывания/ метаболизма витамина D.

\section{Сопоставление с другими публикациями}

Диагностика дефицита витамина D, согласно действующим национальным, а также международным клиническим рекомендациям, основана на определении уровня 25(OH)D в сыворотке крови, и адекватными принято считать уровни 25(OH)D $\geq 30$ нг/мл; уровень 25(OH)D в сыворотке крови <20 нг/мл расценивается как дефицит витамина D, диапазон 20-29 нг/мл трактуется как недостаточная обеспеченность организма витамином [1, 2]. В РФ проведен ряд исследований, результаты которых согласуются с мировыми данными и подтверждают повсеместную распространенность низких уровней витамина D среди населения страны, которые встречаются с частотой от 56 до 100\% [3]. В связи с этим 90\% распространенность дефицита и недостаточности у практически здоровых лиц в нашем исследовании с медианой 25(OH)D 18,8 нг/мл даже в период отсутствия неблагоприятного сезонного влияния дефицита инсоляции не является удивительной.

Среди практически здоровых лиц выявлена высокая частота вторичного гиперпаратиреоза - 30\%, что является лабораторным подтверждением декомпенсации кальций-фосфорного обмена на фоне дефицита витамина D, что также доказывается наличием отрицательной корреляции между 25(OH)D и ПТГ. В генезе вторичного гиперпаратиреоза участвует в том числе кальций $[4,5]$. Низкий уровень потребления кальция с пищей требуется для поддержания кальций-фосфорного обмена, более интенсивной активации витамина D в почках под действием ПТГ, а также стимуляции остеокластов по резорбции костной ткани и, таким образом, мобилизации кальция для общей циркуляции. Влияние низких уровней 25(OH)D на костный метаболизм подтверждается наличием высокой степени корреляции витамина D и уровней CTX, основного показателя интенсивности процессов резорбции, опосредован- ных функцией остеокластов [6]. В данном исследовании мы не оценивали количество потребляемого кальция, что несколько ограничивает интерпретацию данных о том, в каком проценте случаев ведущим фактором является именно низкий уровень витамина D.

Сахарный диабет 2 типа характеризуется нарушением функции $\beta$-клеток поджелудочной железы, инсулинорезистентностью, высокой частотой ожирения и системным воспалением, и есть данные, что витамин D модулирует эти механизмы [7]. Описана потенциальная роль аномального статуса витамина D в нарушении углеводного обмена. В исследованиях на животных и людях было продемонстрировано, что дефицит витамина D пагубно влияет на синтез и секрецию инсулина [8]. В настоящем исследовании мы получили статистически значимо более низкие уровни витамина D в крови относительно практически здоровой популяции - медиана 14,8 нг/мл у лиц с наличием сахарного диабета 2 типа. При этом декомпенсация кальций-фосфорного обмена в виде вторичного гиперпаратиреоза отмечалась у 18,2\%, что статистически не отличалось от частоты этого нарушения в группе контроля. Характерная обратная зависимость 25(OH)D и ПТГ была более выражена, чем в группе контроля ( $r=-0,60$ vs $\mathrm{r}=-0,31)$. Эти данные по высокой распространенности дефицита витамина D у лиц с сахарным диабетом 2 типа подтверждают данные проведенных ранее исследований [9], что может быть следствием высокой частоты ожирения у пациентов данной группы, что обуславливает снижение уровня витамина D как вследствие объемного разведения (количество циркулирующей крови увеличено у лиц с ожирением), так и частичной секвестрации витамина D в адипоцитах $[10,11]$. Коррекция же уровня витамина D у лиц с сахарным диабетом 2 типа подтверждает положительный эффект витамина D на углеводный обмен и уменьшение системного/сосудистого воспаления [12].

ПГПТ и дефицит витамина D - частые состояния из-за широкого внедрения лабораторного тестирования на паратгормон, кальций и 25(OH)D. Многие исследования демонстрируют высокую распространенность дефицита витамина D при ПГПТ, что также продемонстрировано и в настоящей работе. Медиана уровня 25(OH)D в крови при ПГПТ была значимо ниже таковой у группы контроля (15,9 vs 18,8 нг/мл). Предполагается, что более низкие уровни витамина D при ПГПТ возникают из-за ускоренного превращения 25-гидроксивитамина D в кальцитриол или в неактивные 24-гидроксилированные соединения [13, 14]. Витамин D при ПГПТ также участвует во многих патофизиологических процессах при этом заболевании, таких как метаболизм липидов и глюкозы, прогрессирование костных и кардиометаболических осложнений [13]. В связи с чем многими профессиональными сообществами включена в клинические рекомендации по гиперпаратиреозу рекомендация по необходимости восполнения дефицита витамина D [15]. Несколько эпидемиологических исследований подтвердили, что нативный витамин D (холекальциферол) хорошо переносится и безопасен для лиц с дефицитом витамина D при ПГПт. Коррекция дефицита снижает гиперпаратгормонемию, не оказывает симптоматического воздействия на кальциурию и, что особенно важно, улучшает костный и функциональный статус пациентов [14]. 
Болезнь Иценко-Кушинга является одним из вариантов эндогенного гиперкортицизма вследствие гиперпродукции адренокортикотропного гормона аденомой гипофиза, кортикотропиномой. Данные ранних работ, посвященных влиянию избытка глюкокортикоидов на метаболизм витамина D, весьма противоречивы, что обусловлено высокой гетерогенностью исследуемых групп. В 1986 г. Kugai и соавт. показали, что среди лиц с синдромом Иценко-Кушинга и выраженной остеопенией наблюдались более высокие значения концентрации 1,25(OH) 2 В в сравнении с пациентами без остеопении и группой контроля; это было объяснено повышенной кальциурией, которая вызывала повышение ПтГ и, соответственно, синтез 1,25(OH) 2 [ [16]. Дефицит витамина D часто выявляется при состояниях гиперкортицизма, причинами чего могут служить ожирение, а также нарушения метаболизма витамина в сторону катаболических реакций, развивающиеся на фоне избытка глюкокортикоидов. Так, в экспериментальных работах дексаметазон повышал почечную экспрессию 24-гидроксилазы, а при назначении преднизолона ингибируется активность 25-гидроксилазы [17, 18]. В нашей работе также подтверждаются более низкие уровни витамина D у пациентов с болезнью ИценкоКушинга, чем у практически здоровых лиц - 14,6 vs 18,8 нг/мл ( $<<0,05)$ с декомпенсацией кальций-фосфорного обмена в сторону вторичного гиперпаратиреоза у практически четверти пациентов, что было сопоставимо с данными группы контроля ( $>>0,05)$. Так же, как и у группы контроля, выявлена отрицательная обратная связь уровня витамина D с уровнем ПтГ $(r=-0,30)$. Отличия в активной фазе заболевания подтверждают роль глюкокортикоидов в реализации дефицита витамина D у пациентов с болезнью Иценко-Кушинга.

Опубликованные результаты экспериментальных работ указывают на то, что гормон роста, стойко повышенный при акромегалии, способен стимулировать выработку 1,25(OH) 2 D. При введении крысам гормона роста в течение 10 дней наблюдались значимое повышение почечной конверсии 25(OH)D в 1,25(OH) 2 р и снижение конверсии в 24,25(OH) $D$ D $[16,19]$. Показано значимое повышение концентрации $1,25(\mathrm{OH})_{2}$ D у здоровых мужчин при терапии фармакологическими дозами гормона роста и в исследованиях у пациентов с акромегалией гипофизарного генеза. Кроме того, несколькими коллективами исследователей было показано, что инсулиноподобный фактор роста 1 типа (ИФР-1) напрямую стимулирует продукцию 1,25(OH), D почечными клетками, независимо от гормона роста, и повышает концентрацию циркулирующего 1,25(OH) $\mathrm{D}_{3}[20,21]$. Таким образом, предполагается, что повышение концентрации 1,25(OH) 2 D при акромегалии имеет ПТГ-независимый характер и наиболее вероятно обусловлено активацией 1а-гидроксилазы, опосредованной высокими уровнями ИФР-1.

В нашей работе все пациенты исследования были в активной фазе заболевания с повышенными уровнями ИФР-1. Медиана уровней 25(OH)D была значимо ниже, чем в группе контроля, - 14,9 vs 18,8 нг/мл, а вторичный гиперпаратиреоз встречался в $9 \%$ случаев, что статистически не отличалось от частоты в группе контроля. Обратная зависимость 25(OH)D и ПТГ также вы- являлась с хорошими показателями наклона кривой $(r=-0,49 ; p=0,02)$, но данных за повышенную активацию витамина D в 1,25(OH) 2 в виде повышенного уровня кальция крови не получено.

Ограничения исследования

Основным ограничением исследования является его ретроспективный дизайн.

\section{выводы}

1. Выявлена высокая распространенность дефицита и недостатка витамина D в группе практически здоровых пациентов в период отсутствия неблагоприятного сезонного влияния дефицита инсоляции.

2. Наличие хронических эндокринных заболеваний, таких как сахарный диабет 2 типа, ПГПТ, болезнь Иценко-Кушинга, акромегалия, связано со значимо более низкими уровнями витамина D в крови.

\section{ЗАКЛЮЧЕНИЕ}

Данные проведенного исследования подтверждают высокую распространенность дефицита и недостаточности витамина D в общей популяции. При эндокринных заболеваниях, таких как сахарный диабет 2 типа, первичный гиперпаратиреоз, болезнь Иценко-Кушинга и акромегалия, выявлена еще большая распространенность низких уровней витамина $D$, что обуславливает необходимость целенаправленного скрининга и при необходимости диетической коррекции и лечебно-профилактических вмешательств. Витамин $D_{3}$ выпускается в различных формах (водный и масляный раствор, капсулы, таблетки) и дозировках.

Представителем нативного витамина D в виде масляного раствора является Детримакс ${ }^{\circledast}$ Актив, содержащий в 1 капле 500 ME холекальциферола. Детримакс ${ }^{\circledast}$ Актив обладает удобным помповым дозирующим устройством, позволяющим точно и быстро отмерить необходимую дозу витамина D. При дозировании нет необходимости переворачивать флакон с препаратом, что снижает возможность его проливания. Помпа-дозатор делает невозможным случайное увеличение дозы, что является существенной проблемой других препаратов, имеющих стандартную пластиковую капельницу. Детримакс ${ }^{\circledast}$ также представлен таблетированными формами, содержащими $1000 \mathrm{ME}$ и $2000 \mathrm{ME}$ витамина $\mathrm{D}_{3}$ в одной таблетке. Таблетки имеют разделительные риски и удобны в применении.

\section{ДОПОЛНИТЕЛЬНАЯ ИНФОРМАЦИЯ}

Источники финансирования. Работа выполнена по инициативе авторов без привлечения финансирования.

Конфликт интересов. Авторы декларируют отсутствие явных и потенциальных конфликтов интересов, связанных с содержанием настоящей статьи.

Участие авторов. Все авторы одобрили финальную версию статьи перед публикацией, выразили согласие нести ответственность за все аспекты работы, подразумевающую надлежащее изучение и решение вопросов, связанных с точностью или добросовестностью любой части работы. 


\section{СПИСОК ЛИТЕРАТУРЫ | REFERENCES}

1. Пигарова Е.А., Рожинская Л.Я., Белая Ж.Е., и др. Клинические рекомендации российской ассоциации эндокринологов по диагностике, лечению и профилактике дефицита витамина D у взрослых // Проблемы эндокринологии. - 2016. - Т. 62. №4. - C. 60-84. [Pigarova EA, Rozhinskaya LY, Belaya JE, et al. Russian Association of Endocrinologists recommendations for diagnosis, treatment and prevention of vitamin D deficiency in adults. Problems of Endocrinology. 2016;62(4):60-84. (In Russ.)]. doi: https://doi.org/10.14341/probl201662460-84

2. Лесняк О.М., Никитинская О.А., Торопцова Н.В., и др. Профилактика, диагностика и лечение дефицита витамина D и кальция у взрослого населения России и пациентов с остеопорозом (по материалам подготовленных клинических рекомендаций) // Научно-практическая ревматология. - 2015. - Т. 53. — №4. C. 403-408. [ Lesnyak OM, Nikitinskaya OA, Toroptsova NV, et al. The prevention, diagnosis, and treatment of vitamin $\mathrm{D}$ and calcium deficiencies in the adult population of russia and in patients with osteoporosis (according to the materials of prepared clinical recommendations). Rheumatol Sci Pract. 2015;53(4):403-408. (In Russ.)] doi: https://doi.org/10.14412/1995-4484-2015-403-408

3. Петрушкина А.А., Пигарова Е.А., Рожинская Л.Я. Эпидемиология дефицита витамина D в Российской Федерации // Ocmeonopoз u ocmeonamuu. - 2018. - T. 21. — №3. C. 15-20. [Petrushkina AA, Pigarova EA, Rozhinskaya LY. The prevalence of vitamin D deficiency in Russian Federation. Osteoporosis and Bone Diseases. 2019;21(3):15-20. (In Russ.)]. doi: https://doi.org/10.14341/osteo10038

4. Пигарова Е.А., Поваляева А.А., Дзеранова Л.К., Рожинская Л.Я. Роль витамина D в профилактике и лечении остеопороза — новый взгляд на известную проблему // Русский медииинский журнал. Медииинское обозрение. - 2019. — Т. 3. — №10-2. — С. 102-106. [Pigarova EA, Povalyaeva AA, Dzeranova LK, Rozhinskaya LYa. Rol' vitamina D v profilaktike i lechenii osteoporoza — novyi vzglyad na izvestnuyu problem. Russkii meditsinskii zhurnal. Meditsinskoe obozrenie. 2019:3(10-2):102-106. (In Russ.)].

5. Пигарова Е.А., Поваляева А.А., Дзеранова Л.К., Рожинская Л.Я. Роль витамина D для профилактики и лечения рахита у детей // Педиатрия. Consilium Medicum. - 2019. — №3. - C. 4045. [Pigarova EA, Povalyaeva AA, Dzeranova LK, Rozhinskaya LYa. Rol'vitamina D dlya profilaktiki i lecheniya rakhita u detei. Pediatriya. Consilium Medicum. 2019;3:40-45. (In Russ.)]. doi: https://doi.org/10.26442/26586630.2019.3.190582

6. Мельниченко Г.А., Белая Ж.Е., Рожинская Л.Я., и др. Федеральные клинические рекомендации по диагностике, лечению и профилактике остеопороза // Проблемы Эндокринологии. 2017. — T. 63. — №6. - C. 392-426. [Mel'nichenko GA, Belaya ZE, Rozhinskaya $L Y$, et al. Russian federal clinical guidelines on the diagnostics, treatment, and prevention of osteoporosis. Problems of Endocrinology. 2018;63(6):392-426. (In Russ.)]. doi: https://doi.org/10.14341/probl2017636392-426

7. Пигарова Е.А., Петрушкина А.А. Неклассические эффекты витамина D // Ocmeonoроз и остеопатиu. - 2017. - T. 20. № 3. - C. 90-101. [Pigarova EA, Petrushkina AA. Non-classical effects of vitamin D. Osteoporosis and Bone Diseases. 2017;20(3):90-101. (In Russ.)]. doi: https://doi.org/10.14341/osteo2017390-101

8. Alvarez JA, Ashraf A. Role of Vitamin D in Insulin Secretion and Insulin Sensitivity for Glucose Homeostasis. Int J Endocrinol. 2010;2010:1-18. doi: https://doi.org/10.1155/2010/351385
9. Song Y, Wang L, Pittas AG, et al. Blood 25-Hydroxy Vitamin D Levels and Incident Type 2 Diabetes. Diabetes Care. 2013;36(5):1422-1428. doi: https://doi.org/10.2337/dc12-0962

10. Walsh JS, Bowles S, Evans AL. Vitamin D in obesity. Curr Opin Endocrinol Diabetes Obes. 2017;24(6):389-394. doi: https://doi.org/10.1097/MED.0000000000000371

11. Wortsman J, Matsuoka LY, Chen TC, et al. Decreased bioavailability of vitamin D in obesity. Am J Clin Nutr. 2000;72(3):690-693. doi: https://doi.org/10.1093/ajen/72.3.690

12. Neyestani TR, Nikooyeh B, Alavi-Majd H, et al. Improvement of vitamin D status via daily intake of fortified yogurt drink either with or without extra calcium ameliorates systemic inflammatory biomarkers, including adipokines, in the subjects with type 2 diabetes. J Clin Endocrinol Metab. 2012;97(6):2005-2011. doi: https://doi.org/10.1210/jc.2011-3465

13. Vélayoudom-Céphise FL, Wémeau JL. Primary hyperparathyroidism and vitamin D deficiency. Ann Endocrinol (Paris). 2015;76(2):153-162. doi: https://doi.org/10.1016/j.ando.2015.03.022

14. Souberbielle JC, Bienaimé F, Cavalier E, Cormier C. Vitamin D and primary hyperparathyroidism (PHPT). Ann Endocrinol (Paris). 2012;73(3):165-169. doi: https://doi.org/10.1016/j.ando.2012.04.008

15. Дедов И.И., Мельниченко Г.А., Мокрышева Н.Г., и др. Первичный гиперпаратиреоз: клиника, диагностика, дифференциальная диагностика, методы лечения // Проблемы Эндокринологии. 2016. — T. 62. — №6. — C. 40-77. [Dedov II, Melnichenko GA Mokrysheva NG, et al. Primary hyperparathyroidism: the clinical picture, diagnostics, differential diagnostics, and methods of treatment. Problems of Endocrinology. 2017;62(6):40-77. (In Russ.)]. doi: https://doi.org/10.14341/probl201662640-77

16. Поваляева А.А., Пигарова Е.А., Дзеранова Л.К., и др. Особенности метаболизма витамина D при гиперкортицизме и акромегалии // Проблемы Эндокринологии. — 2019. T. 65. - №6. - C. 444-450. [Povaliaeva AA, Pigarova EA, Dzeranova LK, et al. Vitamin D metabolism in hypercorticism and acromegaly. Problems of Endocrinology. 2020;62(6):40-77. (In Russ.)]. doi: https://doi.org/10.14341/probl12099

17. Kurahashi I, Matsunuma A, Kawane T, et al. Dexamethasone Enhances Vitamin D-24-Hydroxylase Expression in Osteoblastic (UMR-106) and Renal (LLC-PK1) Cells Treated with 1a,25-Dihydroxyvitamin D. Endocrine. 2002;17(2):109-118. doi: https://doi.org/10.1385/ENDO:17:2:109

18. Khomenko AV. Cholecalciferol hydroxylation in rat hepatocytes under the influence of prednisolone. Ukr Biochem J. 2013;85(3):90-95. doi: https://doi.org/10.15407/ubj85.03.090

19. Fontaine O, Pavlovitch H, Balsan S. 25-hydroxycholecalciferol metabolism in hypophysectomized rats. Endocrinology. 1978;102(6):1822-1826 doi: https://doi.org/10.1210/endo-102-6-1822

20. Shah R, Licata A, Oyesiku NM, loachimescu AG. Acromegaly as a cause of 1,25-dihydroxyvitamin D-dependent hypercalcemia: case reports and review of the literature. Pituitary. 2012;15(S1):17-22. doi: https://doi.org/10.1007/s11102-010-0286-8

21. Condamine L, Menaa C, Vztovsnik F, et al. Local action of phosphate depletion and insulin-like growth factor 1 on in vitro production of 1,25-dihydroxyvitamin D by cultured mammalian kidney cells. J Clin Invest. 1994;94(4):1673-1679. doi: https://doi.org/10.1172/JCl117512

ИНФОРМАЦИЯ ОБ АВТОРАХ [AUTHORS INFO]:

*Пигарова Екатерина Александровна, д.м.н. [Ekaterina A. Pigarova, MD, PhD]; адрес: Россия, 117036, Москва, ул. Дм. Ульянова, д. 11 [address: 11 Dm. Ulyanova street, 117036 Moscow, Russia]; ORCID: https://orcid.org/0000-0001-6539-466X; eLibrary SPIN: 6912-6331; Scopus Author ID 55655098500; Researcher ID T-9424-2018; e-mail: kpigarova@gmail.com

Дзеранова Лариса Констаниновна, д.м.н. [Larisa K. Dzeranova, MD, PhD]; ORCID: https://orcid.org/0000-0002-0327-4619; eLibrary SPIN: 2958-5555; e-mail: dzeranovalk@yandex.ru

*Автор, ответственный за переписку / Corresponding author. 


\section{ЦИТИРОВАТЬ:}

Пигарова Е.А., Дзеранова Л.К. Высокая распространенность низких уровней витамина D при эндокринных заболеваниях // Ожирение и метаболизм. - 2021. - Т. 18. — №4. - C. 398-405. doi: https://doi.org/10.14341/omet12799 TO CITE THIS ARTICLE:

Pigarova EA, Dzeranova LK. High prevalence of low vitamin D levels in endocrine disorders. Obesity and metabolism. 2020;18(4):398-405. doi: https://doi.org/10.14341/omet12799 